\title{
A statistical and event study of magnetotail dipolarization fronts
}

\author{
D. Schmid ${ }^{1,2}$, M. Volwerk ${ }^{1}$, R. Nakamura ${ }^{1}$, W. Baumjohann ${ }^{1}$, and M. Heyn ${ }^{2}$ \\ ${ }^{1}$ Space Research Institute, Austrian Academy of Sciences, 8042 Graz, Austria \\ ${ }^{2}$ Institute for Theoretical Physics, Technical University Graz, 8010 Graz, Austria
}

Received: 8 February 2011 - Revised: 12 August 2011 - Accepted: 26 August 2011 - Published: 8 September 2011

\begin{abstract}
A study of dipolarization fronts of the Earth's magnetotail has been performed using seven years (20012007) of Cluster data. Events both with and without highspeed earthward flows are included. A superposed epoch analysis of the data shows that the dipolarization is preceeded by a decrease of $B_{\mathrm{Z}}$ before the increase. The duration of the dipolarization tends to be decreasing with increasing velocity of the plasma flows. The thickness of the dipolarization front is on average 1.8 plasma inertial lengths, independent of the plasma velocity. We find that the events fall into two categories: Earthward and tailward moving dipolarizations. The dipolarization fronts can be assumed to be tangential discontinuities and the currents on the front have mainly a perpendicular component.
\end{abstract}

Keywords. Electromagnetics (Plasmas) - Magnetospheric physics (Magnetotail; Storms and substorms)

\section{Introduction}

Magnetotail dipolarizations are usually associated with substorms (e.g. Baumjohann et al., 1999), after reconnection has taken place on the stretched field lines, the newly connected field lines will move towards the Earth, releasing the magnetic tension. This creates fast earthward flows (see e.g. Angelopoulos et al., 1992; Baumjohann et al., 2002) and a turning of the magnetic field from the $\mathrm{x}$-direction along the tail axis into the $\mathrm{z}$-direction perpendicular to the current sheet in the tail, making it look like a more dipole-like field, hence the name dipolarization.

Note that, although true dipolarization only occurs around geosynchronous distances, this term has been expanded in

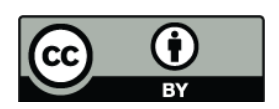

Correspondence to: D. Schmid (schmd@sbox.tugraz.at) meaning to include processes that occur in the Earth's magnetotial. In order to keep nomenclature consistent with the many magnetotail papers that have been published over the last years (cited further below) we use terms dipolarization and dipolarization front for the (reconnection) flowassociated magnetic field turnings.

These dipolarizations still leave a lot of questions open, with respect to the details of their structure like: What is the thickness of the dipolarization front, defined as the quick turning from $B_{\mathrm{x}}$ to $B_{\mathrm{z}}$ ?; Are there currents flowing on the front, and if so, what is the nature of these currents?; Is the structure of the dipolarization front dependent on its velocity?; Why can $B_{\mathrm{Z}}$ decrease to become negative before it starts to increase? In this paper we will try to answer some of these open questions after a short discussion on what has been done before.

Single-spacecraft statistical studies and overall characteristics of fast flows and dipolarizations were obtained, using observations by Geotail (Ohtani et al., 2004) and data from Wind (Sigsbee et al., 2005). The important characteristics obtained in these two papers for this present study are:

1. The magnetic field becomes dipolar in the course of the fast earthward flow;

2. Sharp dipolarization tends to be preceded by a transient decrease in $B_{\mathrm{Z}}$, which starts along with the fast flow and is accompanied by an increase in the plasma density;

3. The plasma and total pressures decrease in the course of the fast flow.

With multi-spacecraft missions one can better determine the characteristics of the dipolarization front. Sergeev et al. (1996) used ISEE 1 and 2 data, measuring a dipolarization of the magnetotail caused by the passage of flux tubes or magnetic bubbles. Nakamura et al. (2002) discussed the motion of a diplarization front. It was found that a slow moving

Published by Copernicus Publications on behalf of the European Geosciences Union. 
front (at $\sim 77 \mathrm{~km} \mathrm{~s}^{-1}$ ) had an estimated size of $\sim 2000 \mathrm{~km}$. A study on the propagation of dipolarizations has been done by Takada et al. (2006), where both Cluster and Double Star data were used. It was found that flow-associated activity disipates within a limited spatial scale $\left(4-8 R_{\mathrm{E}}\right)$ and that the initial topology of the inner magnetosphere contributes strongly to how far fast flows can penetrate towards the Earth (see e.g. Dubyagin et al., 2011).

The THEMIS mission (Angelopoulos, 2002), designed to look at the time-history of substorms was used to show the Earthward flowing of a dipolarization front from $\sim 20 R_{\mathrm{E}}$ to $\sim 11 R_{\mathrm{E}}$ at a velocity of $\sim 300 \mathrm{~km} \mathrm{~s}^{-1}$. Using the passagetime of the front over the spacecraft it was estimated that the thickness of the front was $\sim 400-500 \mathrm{~km}$, i.e. at the size of the ion inertial length, with the larger size found closer to the Earth (Runov et al., 2009).

Li et al. (2011) studied the force balance around dipolarization fronts within bursty bulk flows by comparing curvature force densities and total pressure gradient force densities ahead of and behind the dipolarization fronts. Indeed plasma acceleration immediatiely after the dipolarization front can be explained by the resultant increased curvature force density.

Dipolarization-associated currents in the magnetotail were studied in a variaty of ways: concerning the substorm current wedge in the near-Earth region (Lui et al., 2008) and further down the tail (Nakai and Kamide, 2000; Volwerk et al., 2008). However, determination of the current specifically associated with the dipolarization front has been done by Zhou et al. (2009), who found a current layer with a thickness of the ion inertial length. Zhang et al. (2011) studied current carriers observed within thin current sheets ahead of and during the passage of earthward-moving dipolarization fronts in the near-Earth plasma sheet using THEMIS measurements. Contributions by both diamagnetic and polarization currents were found.

However, in this paper we will use the data from Cluster and use multi-spacecraft analysis to obtain the characteristics of the dipolarization fronts such as thickness and associated currents. The special set-up of the Cluster spacecraft, i.e. in a tetrahedron-shape in regions of interest (e.g. the magnetotail) gives the possiblity of determining the currents through the gradients in the magnetic field (Harvey, 1998).

\section{Data set and selection criteria}

For the event selection, we used the 4-s averaged magnetic field data obtained by the Flux Gate Magnetometer (FGM, Balogh et al., 2001) and plasma data from the Composition Ion Spectrometer (CIS, Rème et al., 2001) on Cluster 1, and throughout the paper the Geocentric Solar Magnetospheric (GSM) coordinate system is used. To survey the dipolarization events in the nightside magnetotail, we used the dataset from Cluster between July and October for the years 2001 to 2007 , when the spacecraft were located between $-20 R_{\mathrm{E}} \leq X_{\mathrm{GSM}} \leq-10 R_{\mathrm{E}}$ and $\left|Y_{\mathrm{GSM}}\right| \leq 15 R_{\mathrm{E}}$.

To find the dipolarization events, we used similar selection criteria as Takada et al. (2006) and Sigsbee et al. (2005). In contrast to these selection criteria we used a 3-min long sliding window instead of a 5-min window. This could result in only "short" dipolarization events, however, it will be shown that there is little effect by the window size. We put on the following requirements on the events:

- The spacecraft is located in the plasma sheet, i.e. plasma- $\beta \geq 0.5$ (Baumjohann et al., 1990).

- The observed earthward plasma flow (perpendicular velocity $V_{\perp}$ in XY plane) is at least in one data point (out of 45 data points in the 3-min window) greater than $V_{\perp, \mathrm{x}} \geq 150 \mathrm{~km} \mathrm{~s}^{-1}$.

- The difference in elevation angle $\theta$ between minimum and maximum $B_{\mathrm{Z}}$ during the window exceeds $\Delta \theta \geq 10^{\circ}$ and $\Delta B_{\mathrm{Z}}$ also exceeds $4 \mathrm{nT}$.

- The elevation angle is at least in one data point greater than $\theta \geq 45^{\circ}$ (Baumjohann et al., 1999).

We have found 355 dipolarization events using Cluster 1 data. Here we restrict the events for mainly earthward dominant flow. That is, we exclude those events for which the flow turns tailward, faster than $-100 \mathrm{~km} \mathrm{~s}^{-1}$ within the 3-min window. From the 355 events 219 events were categorized as earthward dominant flow. This means that there is a large percentage (38\%) of tailward flows with $V_{\mathrm{x}}<-100 \mathrm{~km} \mathrm{~s}^{-1}$ when one compares this number with what is cited by Angelopoulos et al. (1994) for tailward BBFs in the same region (7\%). However Angelopoulos et al. (1994) set a higher velocity limit for tailward BBFs $\left(|V|>400 \mathrm{~km} \mathrm{~s}^{-1}\right)$. Nakamura et al. (2004) found that $22 \%$ of the fast flows, with $|V|>300 \mathrm{~km} \mathrm{~s}^{-1}$, observed in their study were tailward. From these 219 events 160 events had only positive velocity values and 59 events were categorized as tailward-mixed earthward flow events. That means that these 59 events have a slight tailward flow $\left(0>V_{\perp, \mathrm{x}}>-100 \mathrm{~km} \mathrm{~s}^{-1}\right)$ within the 3 -min interval. As the majority of flows in the dataset is categorized as earthward dominant flow, only these 219 events will be investigated further.

To maintain that the spacecraft remains near the center of the plasma sheet, close to the neutral sheet, we require additionally: $\left|B_{\mathrm{x}}\right|<5 \mathrm{nT}$ during the 3-min window. We also set the condition: $\left|Y_{\mathrm{GSM}}\right| \leq 12 R_{\mathrm{E}}$. Consequently 107 events remained for further analysis.

Figure 1 shows the positions of Cluster for the 107 dipolarization events in the GSM XY and YZ plane. As stated above, the dipolarization is preceeded by a decrease in $B_{Z}$ and sometimes this decrease is so strong that $B_{\mathrm{Z}}$ becomes negative. Out of these 107 events, 69 events had positive 
minimum $B_{\mathrm{Z}}$ values during the dipolarization (marked by circles in Fig. 1) and 38 events had negative minimum $B_{\mathrm{z}}$ values (marked by stars in Fig. 1). In the following we examine these 107 events that took place close to the neutral sheet.

\section{Observations}

In this section the changes in the magnetic field $B_{\mathrm{Z}}$ associated with the plasma flow are investigated. Superposed epoch analyses are conducted, where the median and the upper and lower quartiles are used to get the general shape of the dipolarizations and the variation therein.

\subsection{Event view}

Figure 2 shows dipolarization events on 29 August 2003 at 13:53 UT and 1 October 2003 at 21:00 UT. The horizontal axis in Fig. 2 covers a 2-min interval centered at $t=0$. This reference time, $t=0$, corresponds to the start time of the sharpest increase in $B_{\mathrm{Z}}$ within the 2-min interval. We will discuss two specific events first, before starting the superposed epoch analysis of all events chosen for this study.

The event on 29 August 2003 (Fig. 2a) shows a sharp dipolarization front where it took about $4 \mathrm{~s}$ to go from $B_{z, \min }$ to $B_{\mathrm{z}, \max }$ (panel d). The magnetic field elevation angle (panel c) to the equatorial plane at Cluster 1 was about $\sim 60^{\circ}$ before the start of the magnetic dipolarization. There seems to be some oscillations in front of the dipolarization, increasing the elevation angle, after which it decreases to a minimum of $\sim 40^{\circ}$ at $t=0$. The elevation angle then increased to a value of $\sim 70^{\circ}$ at $t=4 \mathrm{~s}$ and then decreases to $\sim 65^{\circ}$. The perpendicular plasma flow is shown in panel $(\mathrm{g})$. The maximum perpendicular earthward flow in x-direction of $\sim 300 \mathrm{~km} \mathrm{~s}^{-1}$ was observed $8 \mathrm{~s}$ after the sharp change of $B_{\mathrm{Z}}$ and decayed gradually $28 \mathrm{~s}$ after the dipolarization. The plasma flow in $\mathrm{y}$ and z-direction was barely existing.

The event on 1 October 2003 (Fig. 2b) shows a dipolarization, where it took about $12 \mathrm{~s}$ to go from $B_{\mathrm{Z}, \min }$ to $B_{\mathrm{z} \text {,max }}$ (panel d). The magnetic field elevation angle to the equatorial plane (panel c) at Cluster 1 was $\sim 50^{\circ}$. The elevation angle first decreased to a minimum of $\sim-20^{\circ}$ and then increased back to a value of $\sim 70^{\circ}$ at $t=20 \mathrm{~s}$. The maximum perpendicular earthward flow (panel g) in $\mathrm{x}$ direction was $\sim 200 \mathrm{~km} \mathrm{~s}^{-1}$ during the dipolarization. The maximum plasma flow in y-direction was significant higher at $\sim 400 \mathrm{~km} \mathrm{~s}^{-1}$ at the dipolarization and decayed gradually $\sim 20 \mathrm{~s}$ after the dipolarization. The plasma flow in z-direction was negligible.

The plasma- $\beta$, shown in panel (a), for both events was 20 and 250 , respectively during the dipolarization, indicating that the spacecraft was well situated in the plasma sheet. The increase in plasma- $\beta$ is caused by increasing the plasma density, $N_{\mathrm{P}}$ (shown in panel b), in front of the dipolarization flow due to the compression of the plasma.
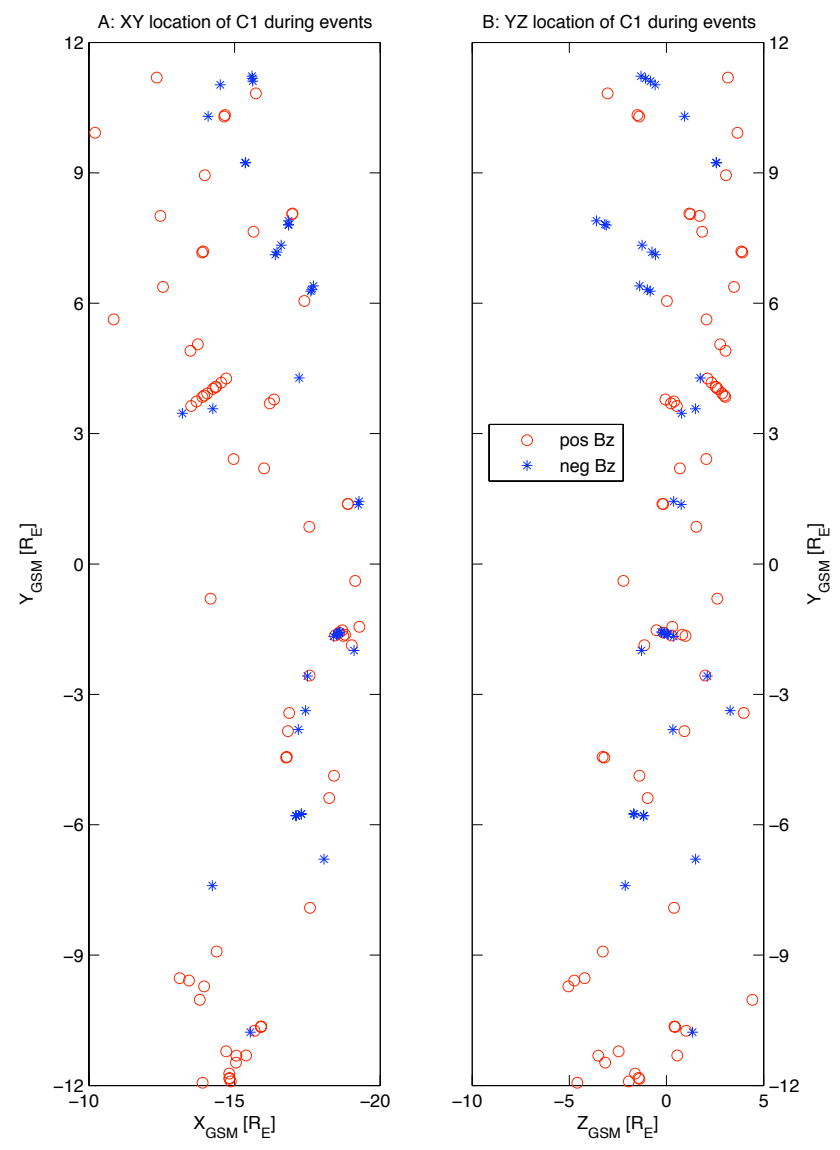

Fig. 1. Cluster spacecraft position in the $X Y$ and $Y Z$ plane for the 107 earthward flow events observed during the years 2001-2007. The 69 events with positive minimum $B_{\mathrm{Z}}$ values are marked by circles. The 38 events with negative minimum $B_{\mathrm{Z}}$ values during the dipolarization are marked by stars.

Panels (h)-(j) in Fig. 2 shows the electric currents determined by the curlometer technique with the currents perpendicular to the magnetic field (shown as filled region under the red curves). However this will be discussed in Sect. 5.

\subsection{Superposed epoch study of dipolarizations}

Figure 3a shows the Z-component of the magnetic field for the 107 dipolarization events $\left(B_{Z}\right.$, black lines) and the median over plotted $\left(\overline{B_{Z}}\right.$, red line). The green lines are the upper and lower quartiles of $\overline{B_{\mathrm{Z}}}$. As one can see, for the median of all these events the $\overline{B_{Z}}$ drops to a minimum before it starts to increase.

To examine how $B_{\mathrm{Z}}$ changes in association with the plasma flow we have divided the dataset into 2 subsets according to the maximum speed of the horizontal perpendicular earthward plasma flow: $150<V_{\perp, \mathrm{xy}}<400 \mathrm{~km} \mathrm{~s}^{-1}$ and $400 \mathrm{~km} \mathrm{~s}^{-1}<V_{\perp, \mathrm{xy}}$. These two subsets are studied with a superposed epoch analysis. In Fig. $3 b$ the relation between the earthward plasma flow velocity $V_{\perp, \mathrm{xy}}$ in the horizontal 

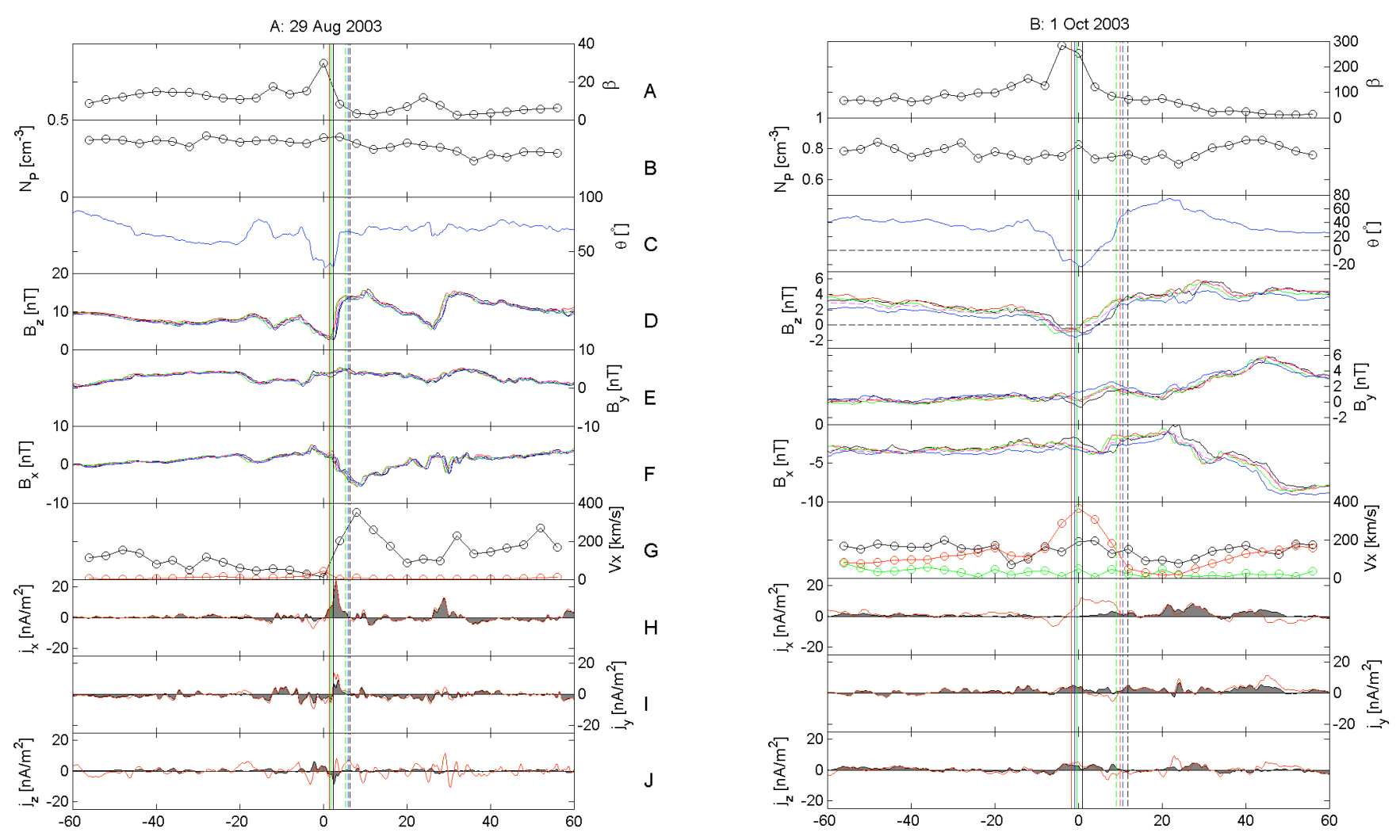

Fig. 2. Data for 29 August 2003 from 13:52 UT till 13:54 UT (left panel) and for 1 October 2003 from 20:59 UT till 21:01 UT (right panel). Panel (A) the proton density; Panel (B) the plasma- $\beta$; Panel (C) the magnetic field inclination angle for C1; Panels (D)-(F) the three magnetic field components for all Cluster spacecraft; Panel G: The perpendicular plasma flow velocity $V_{\mathrm{x}}$ (black), $V_{\mathrm{y}}$ (red) and $V_{\mathrm{z}}$ (green); Panels (H)(J) the three components of the electric currents determined by the curlometer technique with the current perpendicular to the magnetic field shown as the filled region under the red curves. The two specific times for the 'timing-method' (marked by the horizontal colored solid lines for $B_{\mathrm{Z}, \min }$ and the dashed lines for $B_{\mathrm{Z}, \max }$ are also shown.

XY plane and the medians of the two superposed $B_{\mathrm{Z}}$ is plotted. The result of the superposed epoch analysis shows that the gradient of the dipolarization increases with enhanced velocity. For the low velocity set we find $\Delta B_{\mathrm{Z}} \approx 3.5 \mathrm{nT}$ in $\Delta t=12 \mathrm{~s}$, whereas for the high velocity set $\Delta B_{\mathrm{Z}} \approx 6 \mathrm{nT}$ in the same time interval. This observational result suggests that the duration of the dipolarization is anti-correlated with the plasma velocity. Also, the variation in $B_{\mathrm{Z}}$ is much greater for the fast flow, a deeper decrease before the dipolarization and a larger end value after the dipolarization. To obtain information about the temporal and spatial scale seperately, it is necessary to use the data not only form Cluster 1 (as up to now) but also from other Cluster spacecraft.

From the 107 dipolarization events, 38 events have an negative $B_{z, \min }$ value, see the negative values at $t=0$ in Fig. $3 \mathrm{a}$. There can be various possible reasons for a negative $B_{\mathrm{z}, \mathrm{min}}$ value, with the spacecraft close to the center of the plasma sheet. Amongst possible others, there are:

1. The magnetotail can be tilted in the $Y Z$ plane during the observed event and the $B_{\mathrm{Z}}$ undershoot becomes negative because of a non-zero $B_{\mathrm{y}}$ component in the tail. In or- der to test this first explanation, the relation between the mean of the magnetic field in y-direction, $\left|B_{\mathrm{y} \text {, mean }}\right|$ and z-direction, $B_{z, \text { mean }}$ of the 38 dipolarization events with a negative $B_{\mathrm{z}, \text { min }}$ (black circles) is shown in Fig. 4 . The

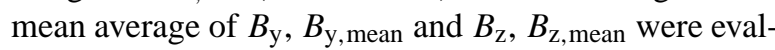
uated during the steady state before the dipolarization occurs.

2. Strong (diamagnetic) currents on the dipolarization front can generate enough disturbance that $B_{\mathrm{Z}}$ can become negative durring the event.

3. A negative $B_{z, \min }$ undershoot can also arise from magnetic flux passing over the SC.

4. Transient reconnection is another possible mechanism producing asymmetric bipolar $B_{\mathrm{Z}}$ variations, as Kiehas et al. (2009) showed. 

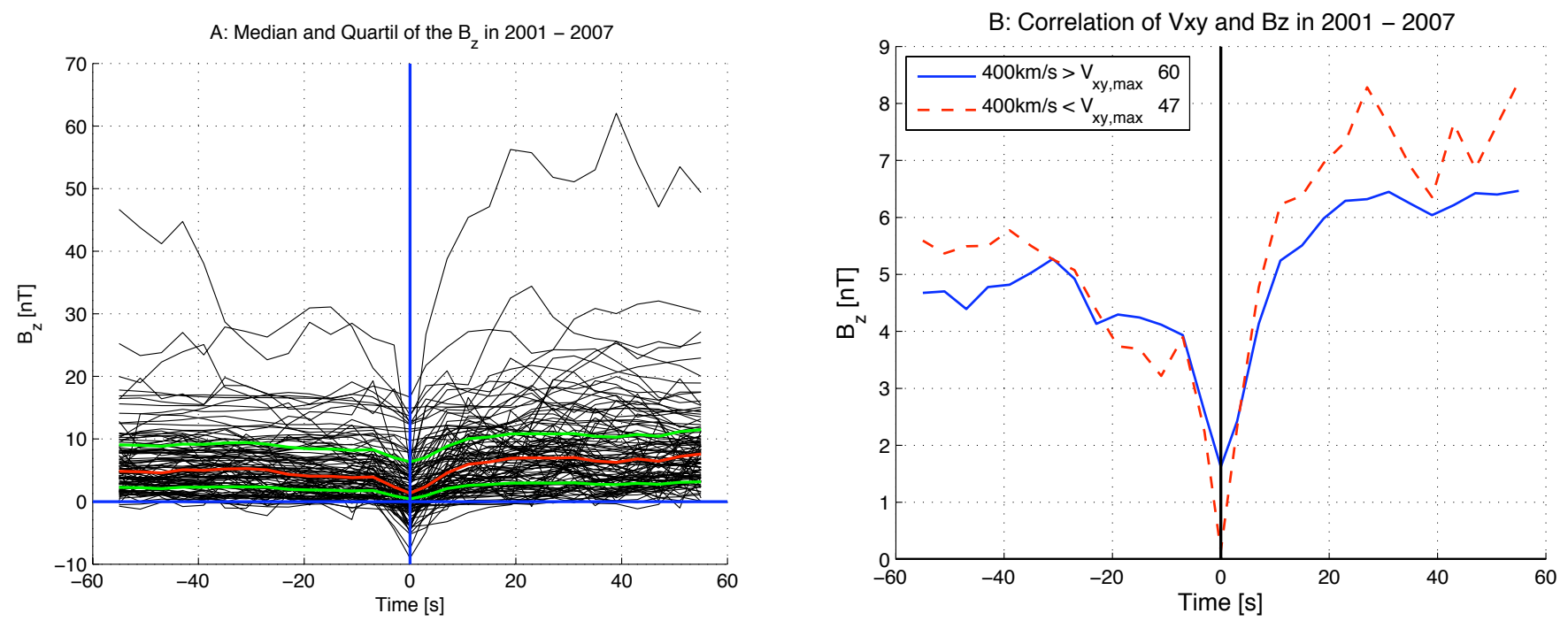

Fig. 3. The left figure shows the $B_{\mathrm{Z}}$ of the 107 dipolarization events (black line) and the median over these events (red line). The green lines are the upper and lower quartile of the median of $B_{\mathrm{Z}}$. The right figure shows the relation between the maximum perpendicular plasma flow velocity in XY plane and the median of the superposed $B_{\mathrm{Z}}$ for the 107 events. From these 107 events 60 events had a maximum velocity in horizontal XY plane $V_{\perp, \mathrm{xy}}<400 \mathrm{~km} \mathrm{~s}^{-1}$ and 47 events a maximum velocity $V_{\perp, \mathrm{xy}}>400 \mathrm{~km} \mathrm{~s}^{-1}$ during the dipolarization.

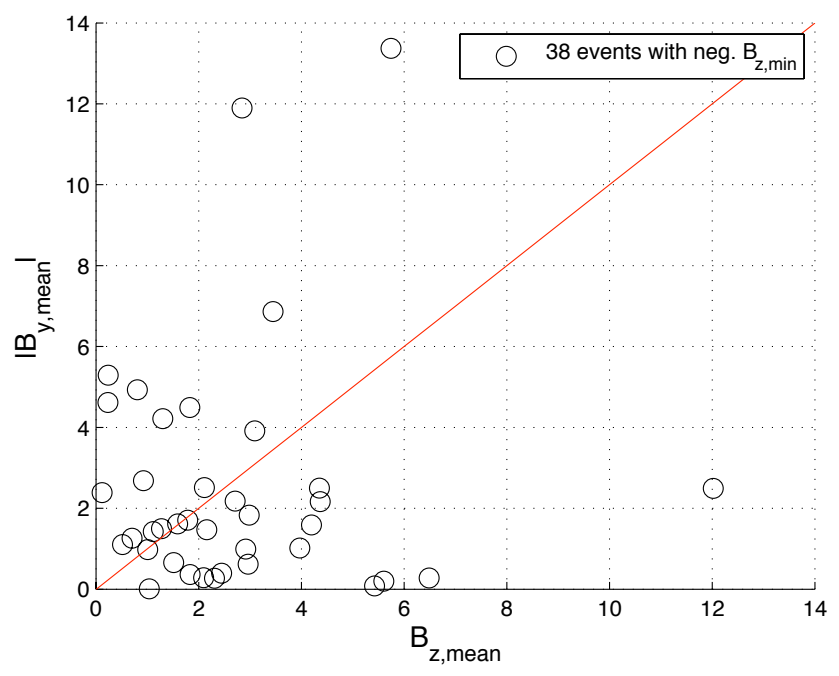

Fig. 4. The relation between the mean of the magnetic field in $y$ direction, $\left|B_{\mathrm{y}, \text { mean }}\right|$ and $\mathrm{z}$-direction, $B_{\mathrm{z} \text {, mean }}$ of the 38 dipolarization events with a negative $B_{\mathrm{z} \text {, min }}$ (black circles). The mean magnitudes are evaluated during the steady state before the dipolarization is about to occur.

\section{Multi-satellite observations}

With the Cluster quartet it is possible to obtain time series at different locations, which can be used to distinguish between the temporal and spatial behavior of the magnetic fields (see e.g. Paschmann and Daly, 1998; Volwerk, 2006). Harvey (1998) showed a simple method to obtain the normal velocity of a plane boundary passing by the Cluster spacecraft, the so called timing-method. Using the spatial configuration of the Cluster spacecraft, one can e.g. cross-correlate the magnetic field data between the spacecraft to obtain the normal velocity of the magnetic structure from which the thickness of the boundary can be inferred.

The timing-method is very sensitive to the resolution of the data. Hence we used 5-Hz resolution data for all 107 dipolarization events which were identified with the 4-s dataset. The high-resolution data are lowpass-filtered to smooth the data and to conserve the profile of the 4-s resolution magnetic field data. The best results was obtained by the use of a lowpass with a cutoff frequency of $f_{\text {cut-off }}=2 \mathrm{~Hz}$. Indeed the profile of the high-resolution magnetic field data had roughly the same profile as the spin resolution magnetic field data, which means that the "high" frequency noise cannot influence the deformation of $B_{\mathrm{z}, \min }$ and $B_{\mathrm{z}, \max }$.

Panels (d)-(f) of Fig. 2 show the magnetic field data of all 4 Cluster spacecraft with 5-Hz data. To obtain the normal velocity of the magnetic structure accurately with the timingmethod, we used two specific times (marked by coloured solid lines ("timing" $B_{z, \min }$ ) and coloured dashed lines ("timing" $\left.B_{z, \max }\right)$ in Fig. $2 \mathrm{~b}$. The particular times are selected automatically by searching the minimum and maximum of $B_{\mathrm{z}}$. Then we estimated the normal velocity of the magnetic structure for each particular time ( $V_{\text {timing,min }}$ and $\left.V_{\text {timing,max }}\right)$. For a first study we are interested in events that are quasistationary, i.e. the velocity does not change too much over the structure, and events that show approximately the same profile to limit spatial variations of the dipolarization within the Cluster tetrahedron. In the following we will call these quasi-stationary events "steady" dipolarization events. 

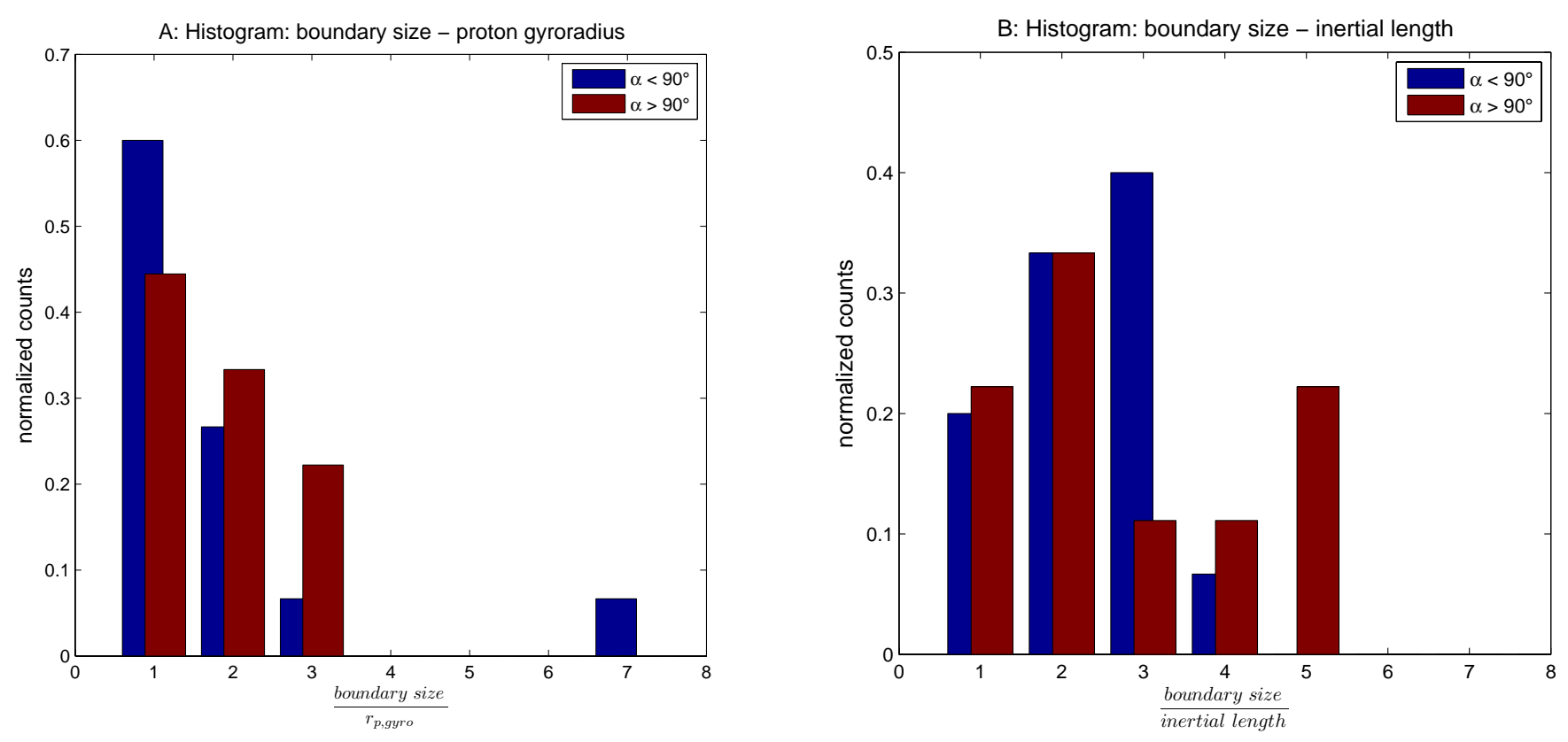

Fig. 5. The left panel (panel A) shows the histogram of the boundary size normalized by the proton gyro radius of the 24 dipolarization events and the right panel (panel B) shows the the histogram of the boundary size normalized by the ion inertial length. 15 events have an angle $\alpha<90^{\circ}$ (marked blue), and 9 events have an angle $\alpha>90^{\circ}$ (marked red). The vertical axis in both plots shows the counts normalized with the total number of events of each group.

The two estimated timing velocities ( $V_{\text {timing,min }}$ and $\left.V_{\text {timing,max }}\right)$ are compared and only events with a velocity difference smaller than $25 \%$ are selected, which we will call "steady" dipolarizations. For further analysis the average of these timing velocities $\left(V_{\text {timing }}=\left(V_{\text {timing, min }}+\right.\right.$ $\left.V_{\text {timing,max }}\right) / 2$ ) was obtained. From the 107 events 66 remained. Another 42 events had a different profile among the four spacecraft based on visual examination of $B_{\mathrm{z}}$. This indicates that there are, indeed, both temporal and spatial variations in the structures associated with the dipolarizations. Therefore, for only 24 events "steady" structure timing velocity could be found. We also obtained the angle $\alpha$ between the estimated normal velocity of the magnetic structure and the maximum perpendicular plasma flow in $\mathrm{XY}$ plane within the 3 -min-long time window. From the 24 events 15 events have an angle $\alpha<90^{\circ}$ (marked by stars in Fig. 6) and 9 events have an angle $\alpha>90^{\circ}$ (marked by triangles in Fig. 6). The estimated angle for the event from 29 August 2003 (Fig. 2a) is $\alpha \approx 30^{\circ}$ and from the event on 1 October 2003 (Fig. 2b) $\alpha \approx 150^{\circ}$. We will discuss the difference between these two flow directions in the discussion section. However, in the following we examine these 24 "steady" dipolarization events.

\subsection{Thickness}

The result of the timing-method for the 24 "steady" dipolarization events confirmed the assumption that the temporal duration of the dipolarization is anti-correlated with the hor- izontal perpendiclular earthward plasma flow velocity $V_{\perp, \mathrm{xy}}$ as shown in Fig. 3. However, there is no systematic behaviour between the thickness of the magnetic boundary $\delta_{\mathrm{D}}$ and the $V_{\perp, \mathrm{xy}} . \delta_{\mathrm{D}}$ was obtained from the time difference between the minimum of $B_{\mathrm{Z}}\left(t_{\text {timing, min }}\right)$ and the maximum of $B_{\mathrm{Z}}\left(t_{\text {timing,max }}\right)$, and the estimated average normal velocity of the magnetic structure from the timing-method $\left(V_{\text {timing }}\right)$ : $\delta_{\mathrm{D}}=\left(t_{\text {timing,max }}-t_{\text {timing,min }}\right) V_{\text {timing. }}$ Like in Sect. $3.2 \mathrm{we}$ split the data into 2 bins based on the maximum $V_{\perp, \mathrm{xy}}: 150$ $400 \mathrm{~km} \mathrm{~s}^{-1}$ and $>400 \mathrm{~km} \mathrm{~s}^{-1}$. From the 24 dipolarization events 11 events have a maximum $V_{\perp, \mathrm{xy}}<400 \mathrm{~km} \mathrm{~s}^{-1}$ and 13 events have a maximum $V_{\perp, \mathrm{xy}}>400 \mathrm{~km} \mathrm{~s}^{-1}$. The obtained average thickness $\delta_{\mathrm{D}}$ of the dipolarization front is $\sim 460 \pm 350 \mathrm{~km}$ and $\sim 430 \pm 350 \mathrm{~km}$ respectively. So the average size of the boundary $(\sim 450 \pm 350 \mathrm{~km})$ seems to be independent of the plasma flow velocity and on the order of the evaluated average proton gyro radius of $\sim 590 \pm 150 \mathrm{~km}$. We also estimated the inertial length of the protons in the magnetic structure: $l_{\text {int }}=\omega_{\mathrm{p}} / c \approx 228 \sqrt{N_{\mathrm{p}}} \mathrm{km}$, where $\omega_{\mathrm{p}}$ is the plasma frequency, $N_{\mathrm{p}}$ is the proton density in $\mathrm{cm}^{-3}$. In additon the relationship between the estimated boundary size and the observed distance from Earth were considered, but no clear correlation was found. Also between the temporal duration of the dipolarization and the observed distance from Earth no clear pattern is found.

In Fig. 5a the histogram of the boundary size normalized by the proton gyro radius is shown and in Fig. $5 \mathrm{~b}$ the histogram of the boundary size normalized by the ion inertial 


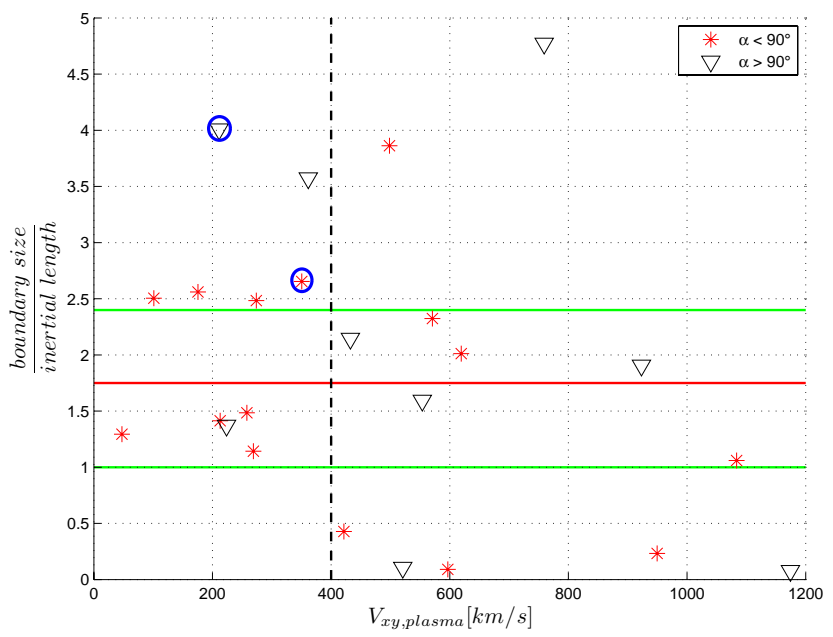

Fig. 6. Ratio of the estimated boundary size of the magnetic structure and the inertial length of the protons in the magnetic structure for the evaluated 24 events. $\alpha$ is the angle between the estimated normal velocity of the magnetic structure and the maximum perpendicular plasma flow in XY plane. The 15 dipolarization events with an angle $\alpha<90^{\circ}$ are marked by stars. The 9 dipolarization events with an angle $\alpha>90^{\circ}$ are marked by triangles. The vertical doted line indicate the 2 velocity bins. The horizontal line is the median of the ratio boundary size and inertial length of the 24 dipolarization events and is about $\sim 1.8$. The horizontal green lines are the upper and lower quartiles of the median. The event from $8 \mathrm{Au}-$ gust 2003 is the star, marked by a circle. The event from 1 October 2003 is the triangle, marked by a circle.

length. From the 24 dipolarization events 15 events have an angle $\alpha<90^{\circ}$ (marked blue), and 9 events have an angle $\alpha>90^{\circ}$ (marked red). Since the total number of events of these two groups is quite different, the vertical axis in both plots has been normalized with the total number of events. For most of the events the estimated thickness of the front is between 1-3 proton gyro radius and independent of $\alpha$. On the other hand the front thickness for most of the events with $\alpha<90^{\circ}$ is less than 3 ion inertial lengths and for the events with $\alpha>90^{\circ}$ no significant information can be obtained.

In Fig. 6 the estimated thicknesses of the dipolarization fronts are scaled to the plasma inertial length. For the 15 events with an angle $\alpha<90^{\circ}$, the ratio $\delta_{\mathrm{D}} / l_{\text {int }}$ is not exceeding a factor of 4 . The median of the ratio of these 15 events is $\sim 1.5$. For the 9 events with an angle $\alpha>90^{\circ}$ three events have a much higher $\delta_{\mathrm{D}} / l_{\text {int }}$ ratio but do not exceed a factor of 5 . The median of the ratio for these 9 events is $\sim 1.9$. The dashed vertical line is the boundary between the two velocity bins. The horizontal red line is the median of the ratio $\delta_{\mathrm{D}} / l_{\text {int }}$ of all 24 dipolarization events and is $\sim 1.8$. The horizontal green lines are the upper and lower quartiles $(\sim 2.5$ and $\sim 1.0$ ). The circles mark the two events from Fig. 2 . The event from 29 August 2003 has a magnetic boundary size of $\sim 970 \pm 240 \mathrm{~km}$, a inertial length of $\sim 370 \pm 60 \mathrm{~km}$ and hence

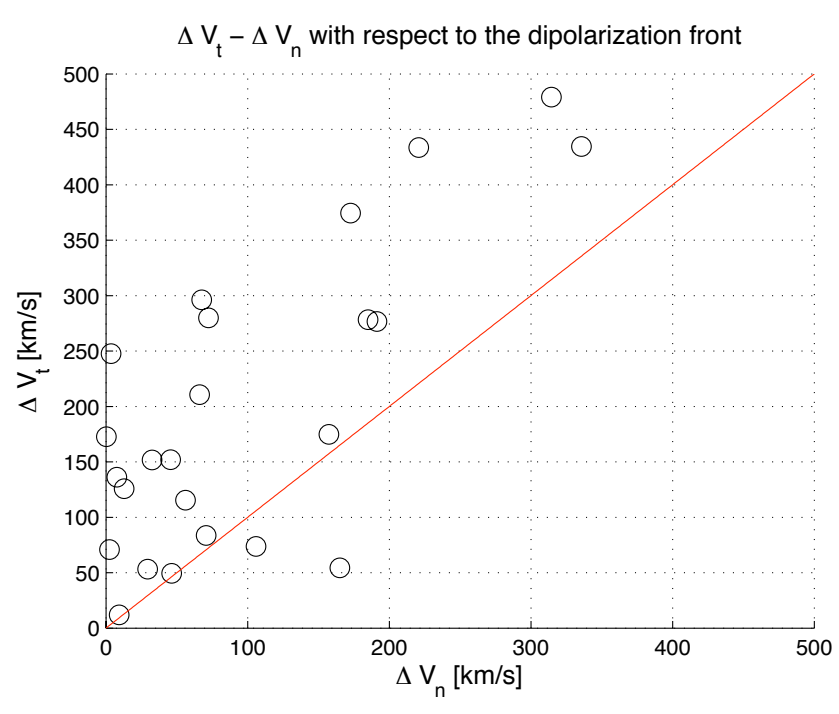

Fig. 7. Relationship between the perpendicular plasma velocity, the estimated timing velocity of the 24 dipolarization events. First we obtained the mean average perpendicular plasma velocity $V_{\perp}$ during the dipolarization (between $B_{\mathrm{z}, \min }$ and $B_{\mathrm{Z}, \max }$ ). Then the velocity difference between the estimated mean plasma velocity and the estimated timing velocity $V_{\text {timing }}$ was determined. Then the velocity difference was projected onto the timing velocity vector and the parallel $\Delta V_{\mathrm{t}}$ and perpendicular components $\Delta V_{\mathrm{n}}$ were evaluated and plotted.

a factor of $\sim 2.5$. The event from 1 October 2003 has a magnetic boundary size of $\sim 1060 \pm 270 \mathrm{~km}$, an inertial length of $\sim 260 \pm 60 \mathrm{~km}$ and hence a factor of $\sim 4$ (see Fig. 6).

Since past studies used the plasma flow velocity in single spacecraft observations to estimate such boundary size (e.g. Runov et al., 2009), a scattered plot of the relationship between the perpendicular plasma velocity, the estimated timing velocity of the 24 events is given in Fig. 7. First we obtained the mean average perpendicular plasma velocity $V_{\perp}$ during the dipolarization (between $B_{z, \min }$ and $B_{\mathrm{z}, \max }$ ). Then the velocity difference between the estimated mean plasma velocity and the estimated timing velocity $V_{\text {timing }}$ was determined. Then the velocity difference was projected onto the timing velocity vector and the parallel $\Delta V_{\mathrm{t}}$ and perpendicular components $\Delta V_{\mathrm{n}}$ were evaluated and plotted. It is often assumed that the dipolarization is a tangential dicontinuity (see e.g. Sergeev et al., 1996), which would mean that $\Delta V$ mainly have tangential component, which is indeed the case for most of the events.

\section{Dipolarization-associated currents}

Any change in magnetic field is associated with current flow and thus it is expected that the dipolarizations found in this paper will also show current signatures. We examine the dipolarization-associated currents on different temporal 


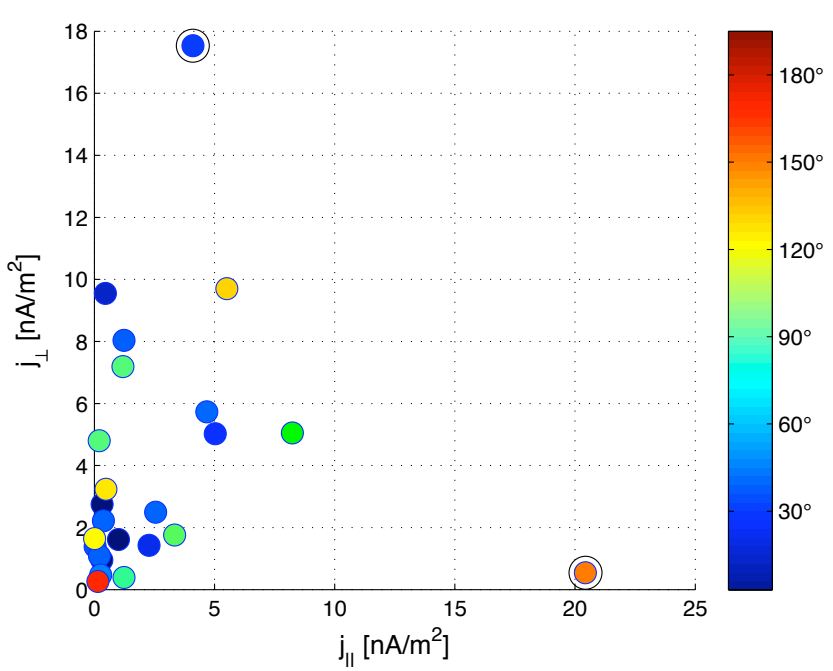

Fig. 8. Scatter plot between the estimated $j_{\perp}$ and $j_{\|}$, each calculated at $B_{\mathrm{Z}, \mathrm{min}}$, with a color scale showing the angle $\alpha$ of the 24 dipolarization events. The event from 29 August 2003 is the blue $\operatorname{dot}\left(\alpha \approx 30^{\circ}\right)$ marked by a black circle and the event from 1 October 2003 is the orange $\operatorname{dot}\left(\alpha \approx 150^{\circ}\right)$ marked by a black circle.

scales. Using the curlometer technique (Harvey, 1998) we can deduce the currents from the magnetic fields measured by Cluster.

Figure 2 (left panel) shows the magnetic field data (panels d-f) for 29 August 2003, where the separation of the spacecraft was $\sim 500 \mathrm{~km}$, and the components of the current are shown too (panels $\mathrm{h}-\mathrm{j}$ ), with the perpendicular part (with respect to the magnetic field at the barycenter of the four spacecraft) shown as a filled region under the curve. On the small scale it can be seen that there is strong perpendicular current in the the x- and y-direction after the minimim in $B_{\mathrm{z}}$, and basically in the dipolarization front. This current is responsible for the decrease in $B_{\mathrm{Z}}$ preceding the dipolarization front. The estimated thickness of the dipolarization front is $\delta_{\mathrm{D}} \approx 970 \mathrm{~km}$ (or $\sim 2.5$ intertial lengths). This means that we are sampling the current on a scale smaller than the structure itself and at near the inertial length.

There is a plasma pressure drop over the dipolarization front from $P_{\mathrm{pl}} \approx 0.4 \mathrm{nPa}$ before to $P_{\mathrm{pl}} \approx 0.2 \mathrm{nPa}$ after the dipolarization front has passed (not shown). This pressure gradient can drive a diamagnetic current through:

$\mathbf{j}_{\mathrm{dia}}=\frac{\mathbf{B} \times \nabla_{\perp} P_{\perp}}{B^{2}}$.

We can estitmate the diamagnetic current assuming: $\nabla_{\perp} p_{\perp} \approx \Delta P_{\mathrm{pl}} / \delta_{\mathrm{D}}$ along the front normal (see e.g. Runov et al., 2011a; Zhang et al., 2011); the normal of the front is determined by timing analysis $\mathbf{n} \approx$ $(0.13,-0.71,0.68)$; the magnetic field at dipolarization maximum $\mathbf{B} \approx(-2,5,14)$ nT. Using Eq. (1) we find for the diamagnetic current $\mathbf{j}_{\text {dia }} \approx(11,3,1) \mathrm{nA} \mathrm{m}^{-2}$. This is in the right direction, as can be seen in Fig. 2a, however, the result is off by a factor of $\sim 2$.

Similarly, we investigate the dipolarization for 1 October 2003, shown in Fig. 2b. This event shows a much slower dipolarization over $\sim 12 \mathrm{~s}$, with a plasma velocity mainly from the z-direction to the $\mathrm{y}$-direction and a timing velocity $\mathbf{V}_{\text {timing }} \approx(-48,-65,55) \mathrm{km} \mathrm{s}^{-1}$, i.e. in the tailward direction, corresponding to a physical size of $\sim 1070 \mathrm{~km}$. The magnetic field at the $B_{\mathrm{Z}}$ peak is $\mathbf{B} \approx(-2,2,4) \mathrm{nT}$. The associated diamagnetic current in this case would be $\mathbf{j}_{\text {dia }}=$ $(44,-10,28) \mathrm{nA} \mathrm{m}^{-2}$ much higher than measured. However, this is a different kind of diplarization as in the previous event as $\alpha>90^{\circ}$. We will investigate this further in the discussion section.

For each of the 24 events we have used the curlometer technique to estimate the current. We have then determined the current parallel and perpendicular to the magnetic field and have produced a scatter plot $j_{\|}$and $j_{\perp}$, taken at the dipolarization front. In Fig. 8 the scatter plot between the estimated $j_{\perp}$ and $j_{\|}$, each calculated at the front, with a color scale showing the angle $\alpha$ is shown. The event from 29 August 2003 is the blue $\operatorname{dot}\left(\alpha \approx 30^{\circ}\right)$ marked by a black circle and the event from 1 October 2003 is the orange $\operatorname{dot}\left(\alpha \approx 150^{\circ}\right)$ marked by a black circle. This shows that the main current on the dipolarization front is perpendicular to the magnetic field and there seems to be no correlation with $\alpha$. Interestingly the 1 October 2003 event shows a strong deviation from the main characteristic of the rest of the currents.

In a recent paper, Runov et al. (2011b) discussed the results of a superposed epoch analysis of dipolarization fronts observed from THEMIS. It was found that the current densites associated with the $B_{\mathrm{Z}}$ increase are on average 5-7 times larger than the current density in the crosstail. This is in good agreement with our results since the estimated $j_{\perp}$ for half of the events is greater than $4 \mathrm{nA} \mathrm{m}^{-2}$ and therefore greater than the nominal crosstail current.

\section{Summary and discussion}

In this paper we studied dipolarization fronts observed by the Cluster satellite at $-20 R_{\mathrm{E}} \leq X_{\mathrm{GSM}} \leq-10 R_{\mathrm{E}}$ and $\left|Y_{\mathrm{GSM}}\right| \leq$ $12 R_{\mathrm{E}}$. Our event selection is based on the perpendicular Earthward plasma flow velocity in x-direction $V_{\perp x}$, the elevation angle $\theta$, and it also requires that the spacecraft is in the plasma sheet close to the neutral sheet.

Superposed epoch analysis was performed to examine the duration of the dipolarization front based on 107 dipolarization events. Utilizing high resolution data from all four Cluster spacecraft, we also evaluated the thickness of the dipolarization based on the four spacecraft timing velocity for 24 diplarization events.

From the statistical study, the main results are summarized as follows: 
1. The duration of the dipolarization front is found to show a tendency to decrease with increasing velocity of the plasma flow.

2. The thickness of the dipolarization front, is found to be independent of the plasma flow velocity and is on average $\sim 450 \pm 350 \mathrm{~km}$.

3. The average size of the dipolarization front is in the order of the evaluated ion gyro radius of $\sim 590 \pm 150 \mathrm{~km}$.

4. The estimated average inertial length of the ions in the magnetic structure is $\sim 340 \pm 60 \mathrm{~km}$ and hence corresponds to the size of the dipolarization front with a factor of $\sim 1.8$.

5. The angle between the Earthward plasma flow direction and dipolarization front motion can either be smaller or greater than $90^{\circ}$, distinguishing between flux transport and flux pile up events.

6. The dipolarization fronts tend to be tangential discontinuities.

7. The currents flowing on the fronts have mainly a perpendicular component to the magnetic field.

The average size for the thickness of the dipolarization front, $450 \mathrm{~km}$, just below the average ion gyro radius and just above the ion inertial length, agrees well with estimates made for case studies by various other authors: Apatenkov et al. (2007) showed a thickness of 400-500 km, whereas Runov et al. (2009) found that the thickness was on the order of the ion inertial length. However, Nakamura et al. (2002) showed a thickness of $2000 \mathrm{~km}$ for one event. The variation in thickness of the events in this paper vary between 0.2 and 4 ion inertial lengths, and it may well be that it depends on parameters that have not yet been taken into account, e.g. the current sheet composition and the current sheet thickness.

Two dipolarization events (out of the 24) were looked at in more detail. These events showed that for one, the Earthward moving dipolarization with $\alpha \approx 30^{\circ}$, the magnetic structure could be well described by the diamagnetic currents flowing on the front. However, for the other event, with tailward moving dipolarization at $\alpha \approx 150^{\circ}$, the estimated diamagnetic current did not fit the data. Indeed the current during this dipolarization is more parallel than perpendicular to the magnetic field. This seems to be a special case in the 24 events that were looked at, as all other events much smaller parallel currents.

The dipolarizations in this study can be divided into two groups, according to the angle between the plasma flow direction and the magnetic field motion direction, with the angle $\alpha$ either smaller or larger than $90^{\circ}$. There is the expected category, in which the plasma and the magnetic field move in the same direction, and the dipolarization that is observed comes from the relaxation of the magnetic tension in the stretched field lines as they move Earthward. These dipolarizations occur when there is unimpeded flow.

The other category is when the plasma flow is Earthward but the dipolarization motion is tailward. This means that there is a pileup of the magnetic field in the tail. One would expect that the plasma flow would be deflected by the barrier which causes the pileup. Indeed, in the event on 1 October 2003 (see Fig. 2b) shows that the plasma flow is indeed deflected towards the dawn side of the tail (large $V_{\mathrm{y}}$ ).

The diamagnetic current that was found for the $29 \mathrm{Au}-$ gust event (see Fig. 2a), was in good agreement with theory, and it flows over an interval of $\sim 4 \mathrm{~s}$, which corresponds to a physical size of $\sim 1000 \mathrm{~km}$, just over 2 times the ion inertial length (see Fig. 6). Zhou et al. (2009) found that the thickness of the current layer was just below one ion inertial length, however, their event happened much closer to the Earth at $X \geq-10 R_{\mathrm{E}}$, where the conditions may be different because of the more dipolar like magnetic field structure.

Further investigation of these structures is necessary and planned to investigate the dependences of the thickness of the dipolarization on various parameters in the Earth's magnetotail and to study the large scale development of these structures in the tail and their possible role in the braking of the associated flow.

Acknowledgements. We would like to acknowledge the Cluster Science Data System (CSDS) and the Cluster Active Archive (CAA).

Guest Editor A. Masson thanks two anonymous referees for their help in evaluating this paper.

\section{References}

Angelopoulos, V.: The THEMIS mission, Space Sci. Rev., 141, 534, 2002.

Angelopoulos, V., Baumjohann, W., Kennel, C. F., Coroniti, R. V., Kivelson, M. G., Pellat, R., Walker, R. J., Lühr, H., and Paschmann, G.: Bursty bulk flows in the inner central plasma sheet, J. Geophys. Res., 97, 4027-4039, 1992.

Angelopoulos, V., Kennel, C. F., Coroniti, F. V., Pellat, R., Kivelson, M. G., Walker, R. J., Russell, C. T., Baumjohann, W., Feldman, W. C., and Gosling, J. T.: Statistical characteristics of bursty bulk flow events, J. Geophys. Res., 99, 21257-21280, 1994.

Apatenkov, S. V., Sergeev, V. A., Kubyshkina, M. V., Nakamura, R., Baumjohann, W., Runov, A., Alexeev, I., Fazakerley, A., Frey, H., Muhlbachler, S., Daly, P. W., Sauvaud, J.-A., Ganushkina, N., Pulkkinen, T., Reeves, G. D., and Khotyaintsev, Y.: Multi-spacecraft observation of plasma dipolarization/injection in the inner magnetosphere, Ann. Geophys., 25, 801-814, doi:10.5194/angeo-25-801-2007, 2007.

Balogh, A., Carr, C. M., Acuña, M. H., Dunlop, M. W., Beek, T. J., Brown, P., Fornacon, K.-H., Georgescu, E., Glassmeier, K.H., Harris, J., Musmann, G., Oddy, T., and Schwingenschuh, K.: The Cluster Magnetic Field Investigation: overview of in-flight performance and initial results, Ann. Geophys., 19, 1207-1217, doi:10.5194/angeo-19-1207-2001, 2001. 
Baumjohann, W., Paschmann, G., and Lühr, H.: Characteristics of high-speed flows in the plasma sheet, J. Geophys. Res., 95, 3801-3809, 1990.

Baumjohann, W., Hesse, M., Kokubun, S., Mukai, T., Nagai, T., and Petrukovich, A. A.: Substorm dipolarization and recovery, J. Geophys. Res., 104, 24995-25000, 1999.

Baumjohann, W., Schödel, R., and Nakamura, R.: Bursts of fast magnetotail flux transport, Adv. Space Res., 30, 2241-2246, 2002.

Dubyagin, S., Sergeev, V., Apatenkov, S., Angelopoulos, V., Runov, A., Nakamura, R., Baumjohann, W., McFadden, J., and Larson, D.: Can flow bursts penetrate into the inner magnetosphere?, Geophys. Res. Lett., 38, L08102, doi:10.1029/2011GL047016, 2011.

Harvey, C. C.: Spatial gradients and volumetric tensor, in: Analysis Methods for Multi-Spacecraft Data, edited by: Paschmann, G. and Daly, P., pp. 307-322, ESA, Noordwijk, 1998.

Kiehas, S. A., Semenow, V. S., Kubyshkina, M., Angelopoulos, V., Nakamura, R., Keika, K., Ivanova, V. V., Biernat, H. K., Baumjohann, W., Mende, S., Magnes, W., Auster, U., Fornacon, K. H., Larson, D., Carlson, C. W., Bonnell, J., and McFadden, J.: First application of a Petschek-type reconnection model with timevarying reconnection rate to THEMIS observations, J. Geophys. Res., 114, A00C20, doi:10.1029/2008JA013528, 2009.

Li, S., Angelopoulos, V., Runov, A., X.-Z-Zhou, McFadden, J., Larson, D., Bonnell, J., and Auster, U.: On the force balance around dipolarization fronts within bursty bulk flows, J. Geophys. Res., 116, A00I35, doi:10.1029/2010JA015884, 2011.

Lui, A. T. Y., Volwerk, M., Dunlop, M. W., Alexeev, I. V., Fazakerley, A. N., Walsh, A. P., Lester, M., Grocott, A., Mouikis, C., Henderson, M. G., Kistler, L. M., Shen, C., Shi, J. K., Zhang, T. L., and Rème, H.: Near-Earth substorm features from multiple satellite observations, J. Geophys. Res., 113, A07S26, doi:10.1029/2007JA012738, 2008.

Nakai, H. and Kamide, Y.: Substorm currents associated with magnetotail magnetic dipolarization: Geotail observations, J. Geophys. Res., 105, 18781-18792, 2000.

Nakamura, R., Baumjohann, W., Klecker, B., Bogdanova, Y., Balogh, A., Rème, H., Bosqued, J. M., Dandouras, I., Sauvaud, J.-A., Glassmeier, K.-H., Kistler, L., Mouikis, C., Zhang, T. L., Eichelberger, H., and Runov, A.: Motion of the dipolarization front during a flow burst event observed by Cluster, Geophys. Res. Lett., 29, 1942, doi:10.1029/2002GL015763, 2002.

Nakamura, R., Baumjohann, W., Mouikis, C., Kistler, L. M., Runov, A., Volwerk, M., Asano, Y., Vörös, Z., Zhang, T. L., Klecker, B., Rème, H., and Balogh, A.: Spatial scale of high-speed flows in the plasma sheet observed by Cluster, Geophys. Res. Lett., 31, L09894, doi:10.1029/2004GL019558, 2004.

Ohtani, S., Shay, M. A., and Mukai, T.: Temporal structure of the fast convective flow in the plasma sheet: Comparison between observations and two-fluid simulations, J. Geophys. Res., 109, 3-8, doi:10.1029/2003JA010002, 2004.

Paschmann, G. and Daly, P.: Analysis Methods for MultiSpacecraft Data, ESA, Noordwijk, 1998.

Rème, H., Aoustin, C., Bosqued, J. M., Dandouras, I., Lavraud, B., Sauvaud, J. A., Barthe, A., Bouyssou, J., Camus, Th., CoeurJoly, O., Cros, A., Cuvilo, J., Ducay, F., Garbarowitz, Y., Medale, J. L., Penou, E., Perrier, H., Romefort, D., Rouzaud, J., Vallat, C., Alcaydé, D., Jacquey, C., Mazelle, C., d'Uston, C., Möbius, E.,
Kistler, L. M., Crocker, K., Granoff, M., Mouikis, C., Popecki, M., Vosbury, M., Klecker, B., Hovestadt, D., Kucharek, H., Kuenneth, E., Paschmann, G., Scholer, M., Sckopke, N., Seidenschwang, E., Carlson, C. W., Curtis, D. W., Ingraham, C., Lin, R. P., McFadden, J. P., Parks, G. K., Phan, T., Formisano, V., Amata, E., Bavassano-Cattaneo, M. B., Baldetti, P., Bruno, R., Chionchio, G., Di Lellis, A., Marcucci, M. F., Pallocchia, G., Korth, A., Daly, P. W., Graeve, B., Rosenbauer, H., Vasyliunas, V., McCarthy, M., Wilber, M., Eliasson, L., Lundin, R., Olsen, S., Shelley, E. G., Fuselier, S., Ghielmetti, A. G., Lennartsson, W., Escoubet, C. P., Balsiger, H., Friedel, R., Cao, J.-B., Kovrazhkin, R. A., Papamastorakis, I., Pellat, R., Scudder, J., and Sonnerup, B.: First multispacecraft ion measurements in and near the Earth's magnetosphere with the identical Cluster ion spectrometry (CIS) experiment, Ann. Geophys., 19, 1303-1354, doi:10.5194/angeo19-1303-2001, 2001.

Runov, A., Angelopoulos, V., Sitnov, M. I., Sergeev, V. A., Bonnell, J., McFadden, J. P., Larson, D., Glassmeier, K.H., and Auster, U.: THEMIS observations of an earthwardpropagating dipolarization front, Geophys. Res. Lett., 36, L14106, doi:10.1029/2009GL038980, 2009.

Runov, A., Angelopoulos, V., Sitnov, M., Sergeev, V., Nakamura, R., Nishimura, Y., Frey, H., McFadden, J., Larson, D., Bonnell, J., Glassmeier, K.-H., Auster, U., Connors, M., Russell, C., and Singer, H.: Dipolarization fronts in the magnetotail plasma sheet, Planet. Space Sci., 59, 517-525, doi:10.1016/j.pss.2010.06.006, 2011a.

Runov, A., Angelopoulos, V., Zhou, X.-Z., Zhang, X.-J., Li, S., Plaschke, F., and Bonnell, J.: A THEMIS multicase study of dipolarization fronts in the magnetotail plasma sheet, J. Geophys. Res., 116, A05216, doi:10.1029/2010JA016316, 2011 b.

Sergeev, V. A., Angelopoulos, V., Gosling, J. T., Cattell, C. A., and Russell, C. T.: Detection of localized, plasma-depleted flux tubes or bubbles in the midtail plasma sheet, J. Geophys. Res., 101, 10817-10826, 1996.

Sigsbee, K., Slavin, J. A., Lepping, R. P., Szabo, A., Øieroset, M., Kaiser, M. L., Reiner, M. J., and Singer, H. J.: Statistical and superposed epoch study of dipolarization events using data from Wind perigee passes, Ann. Geophys., 23, 831-851, doi:10.5194/angeo-23-831-2005, 2005.

Takada, T., Nakamura, R., Baumjohann, W., Asano, Y., Volwerk, M., Zhang, T. L., Klecker, B., Rème, H., Lucek, E. A., and Carr, C.: Do BBFs contribute to inner magnetosphere dipolarizations: Concurrent Cluster and Double Star observations, Geophys. Res. Lett., 33, L21109, doi:10.1029/2006GL027440, 2006.

Volwerk, M.: Multi-Satellite observations of ULF waves, in: Magnetospheric ULF waves: Synthesis and new directions, edited by: Takahashi, K., Chi, P. J., Denton, R. E., and Lysak, R. L., pp. 109-135, AGU, Washingtong, 2006.

Volwerk, M., Lui, A. T. Y., Lester, M., Walsh, A. P., Alexeev, I., Cao, X., Dunlop, M. W., Fazakerley, A. N., Grocott, A., Kistler, L., Lun, X., Mouikis, C., Pu, Z., Shen, C., Shi, J. K., Taylor, M. G. G. T., Baumjohann, W., Nakamura, R., Runov, A., Vörös, Z., Zhang, T. L., Takada, T., Rème, H., Klecker, B., and Carr, C. M.: Magnetotail dipolarization and associated current systems observed by Cluster and Double Star, J. Geophys. Res., 113, A08S90, doi:10.1029/2007JA012729, 2008.

Zhang, X.-J., Angelopoulos, V., Runov, A., Zhou, X.-Z., Bonnell, J., McFadden, J. P., Larson, D., and Auster, U.: 
Current-carriers near dipolarization fronts in the magnetotail: A THEMIS event study, J. Geophys. Res., 116, A00I20, doi:10.1029/2010JA015885, 2011.
Zhou, M., Ashour-Abdalla, M., Deng, X., Schriver, D., ElAlaoui, M., and Pang, Y.: THEMIS observation of multiple dipolarization fronts and associated wave characteristics in the near-Earth magnetotail, Geophys. Res. Lett., 36, L20107, doi:10.1029/2009GL040663, 2009. 